\section{Risikobewertung von Patienten mit CTEPH}

Delcroix $\mathrm{M}$ et al. Risk assessment in medically treated chronic thromboembolic pulmonary hypertension patients. Eur Respir J 2018; doi:10.1183/13993003.00248-2018

Mit der von der European Society of Cardiology (ESC) und European Respiratory Society (ERS) entwickelten Beurteilungsstrategie für Patienten mit pumonaler Hypertonie (PH) sind genaue Risikoeinteilungen möglich. Delcroix et al. untersuchten, ob eine verkürzte Version der ESC/ERSRisikobewertung auch für Patienten mit inoperabler chronisch-thromboembolischer pulmonaler Hypertonie (CTEPH) anwendbar ist.

Als Datenbasis dieser Studie diente das Register für pulmonale Hypertonie COMPERA. Von den darin erfassten Variablen verwendeten die Autoren 6 aus, mit denen sie die gekürzte ESC/ERS-Risikobewertung validierten: WHO-Funktionsklasse, 6-Minuten-Gehtest, natriuretisches Peptid Typ B (BNP), rechtsatrialer Druck (RAP), Herzindex (HI) und gemischtvenöse Sauerstoffsättigung $\left(\mathrm{SvO}_{2}\right)$. Die ausgewählten Patienten mussten folgende Kriterien erfüllen:

- therapienaiv mit neu diagnostizierter CTEPH oder residueller $\mathrm{PH}$ nach pulmonaler Endarteriektomie sowie einer Ausgangs- und mindestens einer Nachuntersuchung

- mittlerer pulmonal-arterieller Druck ( $\mathrm{mPAP} \geq 25 \mathrm{mmHg}$ ) und pulmonalkapillärer Verschlussdruck (PAWP $\leq 15 \mathrm{mmHg}$ ) zum Zeitpunkt der Diagnose

- keine pulmonale Endarteriektomie oder pulmonale Ballonagioplastie während der Nachbeobachtung

- $\geq 3$ der 6 Variablen bei der Ausgangsuntersuchung vorliegend

Insgesamt 561 Patienten erfüllten die Einschlusskriterien. Von 231 Patienten standen Nachuntersuchungsdaten mit $\geq 3$ von 6 der geforderten Variablen zur Verfügung. Bei ihnen lag die Zeit zwischen Studieneinschluss und Risikobewertung in der Nachbeobachtung bei median 7 Monaten. In der Nachbeobach- tungszeit ab der Diagnose von bis zu 5 Jahren starben insgesamt 132 Patienten, $46,9 \%$ in der Hoch-, $20,9 \%$ in der mittleren Risiko- und 7,4\% in der Niedrigrisikogruppe.

\section{Signifikante Risikounter- scheidung}

Die zu Beginn der Beobachtung geschätzten 1- und 5-Jahresüberlebensraten unterschieden sich signifikant $(p<0,0001)$ innerhalb der einzelnen Risikogruppen:

- niedriges Risiko. 98,6\% (1-Jahresüberleben), 88,3\% (5-Jahresüberleben)

- mittleres Risiko: 94,9\% (1-Jahresüberleben), 61,8\% (5-Jahresüberleben)

- hohes Risiko: 75,5\% (1-Jahresüberleben), 32,9\% (5-Jahresüberleben)

Die 1-Jahresüberlebensraten wiesen mit $100 \%$ (niedriges Risiko), 91,6\% (mittleres Risiko) und $69,4 \%$ noch deutlichere Unterschiede auf.

\section{FAZIT}

Die verkürzte Version der ESC/ERSRisikobewertung könnte nach diesen Ergebnissen für Patienten mit CTEPH angewendet werden. Sie ermöglichte eine Risikostratifizierung der 5-Jahresmortalität zu Studienbeginn und in der Nachbeobachtung. Die Risikoabschätzungen der ESC/ERS-Empfehlungen für PH-Patienten wurden bestätigt.

Matthias Manych, Berlin 